\title{
EFFECT OF GAP ON THE FLOW CHARACTERISTICS IN THE WAKE OF A BLUFF BODY NEAR A WALL
}

\author{
G. NASIF, R. BALACHANDAR, \& R.M. BARRON \\ University of Windsor, Ontario, Canada.
}

\begin{abstract}
A numerical investigation is carried out to evaluate the influence of the gap between the bluff body and the bed on the wake characteristics generated in shallow flows. A sharp-edge bluff body with a fixed gap from the bed is employed in the study, and the results are compared with the no gap case. A sharp-edged bluff body was chosen to minimize the effect of Reynolds number and ensure fixed flow separation points. The transient three-dimensional Navier-Stokes equations are numerically solved using a finite volume approach with the detached eddy simulation turbulence model. The flow field in this study involves two different fluids, i.e. water and the air above it. The volume of fluid method is used for tracking the free surface separating the water and air. The fluid structures that are generated in the wake are identified using the $\lambda_{2}$-criterion. The results reveal that the gap flow will develop a new structure near the bed, which enhances the upwash flow immediately after the submerged jet is about to turn upwards due to the weak hydraulic jump. This structure plays an important role in recovering the free surface to its original shape at a shorter downstream distance from the bluff body than when there is no gap.

Keywords: free surface, Navier-Stokes equation, numerical simulation, turbulent models, two-phase flow, viscous flow, wake flow, wall jet.
\end{abstract}

\section{INTRODUCTION}

In shallow flow, the clearance between the ground and obstacles can considerably influence the wake flow characteristics. Examples of this type of flow are often found in engineering applications, e.g. the airflow around solar panels, flow around vehicles and the cooling of electronic components. Many investigations have been carried out with circular and square cylinders as a bluff body to examine the influence of the gap on the wake characteristics [1-5]. All these studies agree that the gap alters the wake characteristics if it is introduced in the wake flow. Shinneeb and Balachandar [6-8] carried out an experimental investigation using PIV to determine the gap height effect on the turbulent shallow wake generated by a vertical sharp-edged flat plate suspended in a shallow channel flow. The study disclosed that the size of the wake in the near-bed locations increases with the increase of the gap height as the gap flow enhances the lateral entrainment. The results also revealed that if the gap flow is weak and there is insufficient momentum to overcome the influence of the recirculation, the flow is engulfed by the recirculation zone formed just behind the bluff body. If the gap flow is relatively strong, it penetrates in the downstream direction, and only a portion of it is deflected upwards to feed the recirculation zone.

The main objective of the current study is to numerically assess the effect of the gap flow between the base of the sharp-edge bluff body and the open channel bed on the fluid structures in the wake at near-bed locations. These structures define the downstream location where the free surface restores its original shape behind the bluff body. The final goal of the numerical study is to provide an enhanced analysis not yet possible from the recent experimental studies. A three-dimensional time-dependent detached eddy simulation (DES) is used as the turbulence model in the study. Available data from previous PIV experiments [6-7] are used to validate the numerical results. 


\section{MODEL SETUP AND COMPUTATIONAL METHOD}

Figure 1a is used as the computational domain to obtain an enhanced picture of the effect of the gap on the wake flow in this study. The incoming flow is a fully developed boundary layer type and is sheared in the vertical direction. This boundary layer type of flow can be destabilized by a sudden change of topology, introduction of horizontal shear or deceleration of the flow. In the present study, the destabilizing (and as a result, the wake) is generated by inserting a vertical sharp-edged plate in the open channel as shown in Fig. 1. The plate of width $D=30 \mathrm{~mm}$ is adjusted vertically to create different clearances with the bed, namely, $h / H=0.0$ and 0.1 , where $h$ is the gap height between the base of the plate and the bed, and $H$ is the height of the water in the channel. The wake flow simulations are performed using the finite volume method with the STAR-CCM+ commercial code [9].

In the current simulations, structured cells were used to mesh the computational domain. The mesh is clustered around the bluff body, near the bed and in the wake region to better capture the bed effect and the various flow features. Many grids were considered in the current study, namely, 10, 15, 20 and $22 \times 10^{6}$ cells. In each case, many parameters were checked and compared with experimental results. Furthermore, successive grids were compared to determine whether or not there was a change in the mean characteristics, wake size and turbulence structures. On this basis, the final computations were performed using $22 \times 10^{6}$ cells. Uniform cells $(2 \mathrm{~mm}$ ) have been used in the grid everywhere in the computational domain except at the wall locations. The homogeneous distribution of cells is employed to overcome difficulties faced in the earlier stage of the study in identifying the flow structures in the transitional regions. A minimum cell size of $0.05 \mathrm{~mm}$ appears adjacent to the wall and the bluff body. Ten layers of fine prism cells packed in a $1.0 \mathrm{~mm}$ width with a stretching factor of 1.5

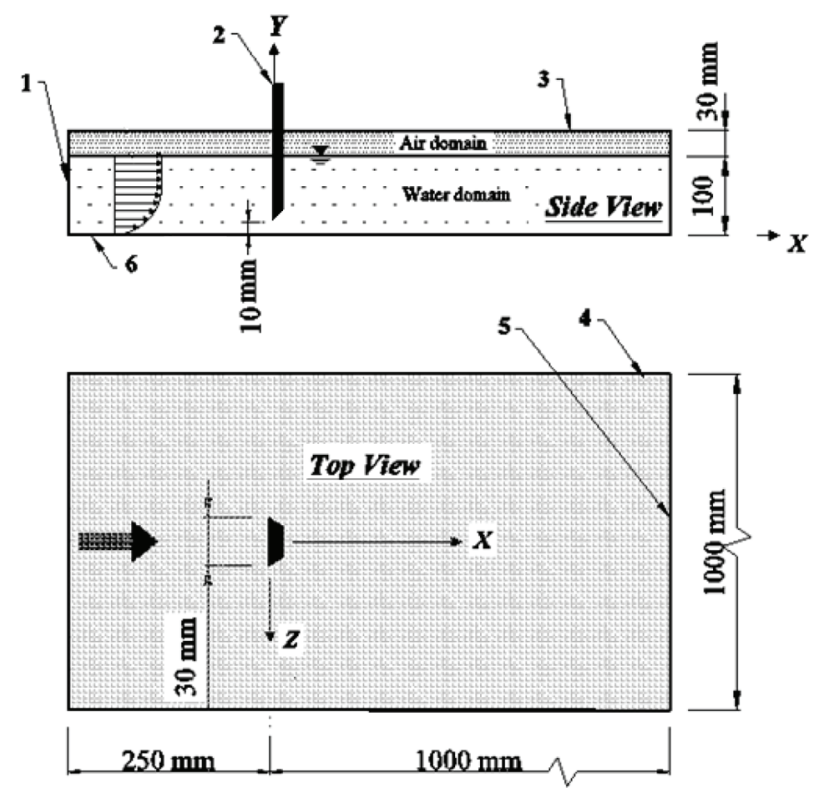

1: Boundary layer velocity profile (inlet); 2 : Sharp-edged bluff body; 3 and 4: Channel ceiling and sides (slip walls); 5: Outlet boundary (atm. pressure); 6: Bed (no-slip wall).

Figure 1: Computational model with appropriate boundary conditions. 
is employed to resolve the wall effect. First-order implicit time marching and second-order spatial differencing are used in the current study to discretize the governing equations. Customarily, the value of $y+(y+=0.4$ in current study) is calculated based on the distance of the centre of the first cell row from the wall.

The volume of fluid (VOF) method suggested by Hirt and Nichols [10] is a free surface modelling technique for tracking the free surface. The VOF model can model two or more immiscible fluids by solving a single set of momentum equations and tracking the volume fraction of each of the fluids throughout the domain. The major complication associated with the VOF method is the smearing of the free surface. This problem originates from extreme diffusion of the transport equation at the interfaces. This issue can be mitigated by using the high-resolution interface capturing (HRIC) scheme [11] to discretize the convective terms in the governing equations. The normalized variable diagram (NVD) [12] is very useful for analysing boundedness properties of convective discretization schemes and provides the methodology used in constructing high-resolution schemes. In high-resolution schemes the local Courant-Friedrichs-Lewy (CFL) condition $(\mathrm{CFL} \leq 1.0)$ should be satisfied to ensure numerical stability. In the current study, different time steps were tested to satisfy the CFL condition and a final time step was set as $1 \times 10^{-3} \mathrm{~s}$. Twenty internal iterations were employed at each time step. The frequency of vortex shedding corresponds to a shedding period $T=0.51 \mathrm{~s}$. The time of $60 \mathrm{~s}$ is used for data averaging, which yields $t_{a v} / T>100$.

DES [13] is a modification of a RANS model which treats near-wall regions in a RANSlike manner, and it treats the rest of the flow in an LES-like manner. The model was originally formulated by replacing the distance function in the Spalart-Allmaras model with a modified distance function. The $k$ - $w$ SST (shear stress transport) turbulence model is a two-equation eddy viscosity model [14-15] and has been selected as the RANS part of the DES turbulence model in this study. The $k$-w SST model solves additional transport equations for turbulent kinetic energy $k$ and specific dissipation rate $w$, from which the turbulent kinematic viscosity $\left(v_{t}=k / w\right)$ can be derived. The transport equations of $k$ and $w$ are described in [16].

Validations are carried out with a no-gap case by comparing the numerical results from the simulations, i.e. mean and statistical features, with available data from previous PIV experiments reported in [6-7]. Figures 2 and 3 show selected samples of the validation process; here $U, W$ and $U_{s}$ represent the streamwise, transverse and the upstream approaching velocity at the given horizontal location from the bed, respectively. The comparisons between the experimental and computational results for the normalized mean quantities and for other statistical quantities (not shown here) reveal that the current model can produce results with adequate accuracy at different elevations from the bed. The model predicts the experimental data with less than $12 \%$ maximum difference occurring in the horizontal plane $y / H=0.1$.

\section{RESULTS AND DISCUSSION}

Nasif et al. [18-20] numerically investigated the three-dimensional fluid structures in the shallow wake flow in the absence of the gap using the $\lambda_{2}$-criterion. In the previous studies, the interactions between the fluid structures, bed, free surface and the bluff body in the mean and transient flow are analysed and addressed. At this point, to understand the effect of the gap flow on the turbulent structures and therefore on the free surface, it is worthwhile to briefly review the turbulent structures that are present in the mean flow from previous investigations for the case with no gap.

Figure 4 shows the three-dimensional fluid structures shaded by the normalized streamwise vorticity $\left(\omega_{x} D / U_{\infty}\right)$ for the case of no gap, where $\omega_{x}$ and $U_{\infty}$ represent the streamwise vorticity and the free-stream velocity, respectively. In this figure, fluid structures are extracted 
(a) $y / H=0.1, x / H=0.1$

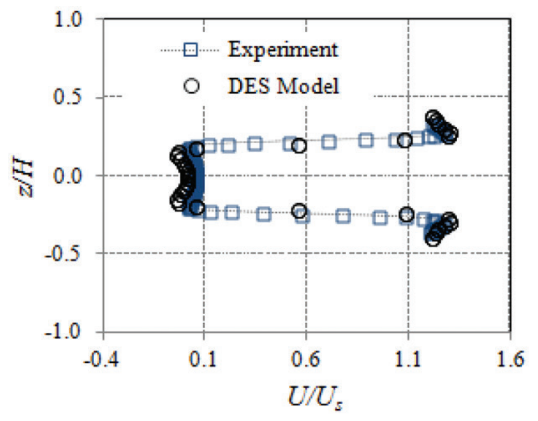

(c) $y / H=0.8, x / H=0.1$

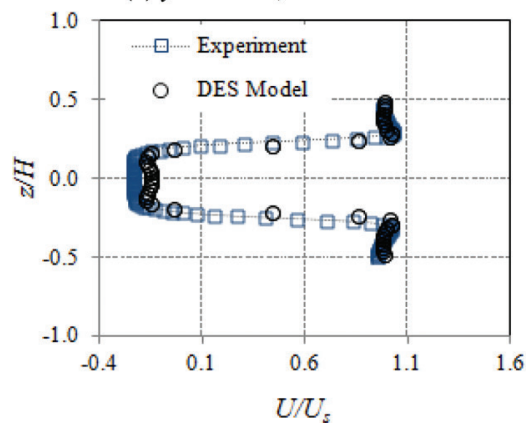

(b) $y / H=0.1, x / H=0.9$

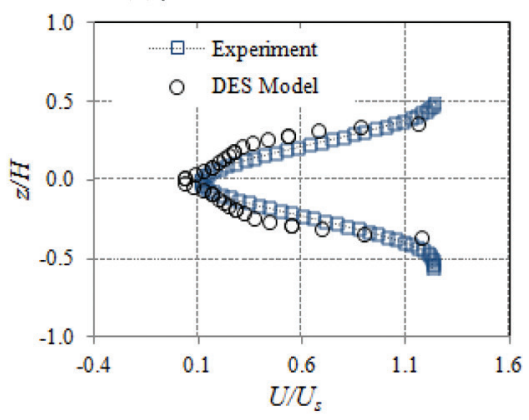

(d) $y / H=0.8, x / H=0.9$

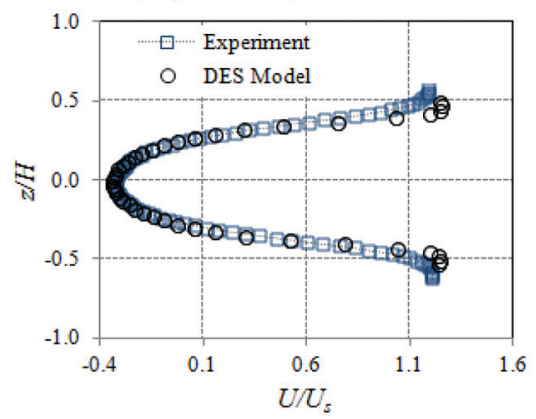

Figure 2: Computational and experimental [6-7] normalized mean streamwise velocity profiles at four different locations in the wake flow [17].

(a) $y / H=0.5, x / H=0.1$

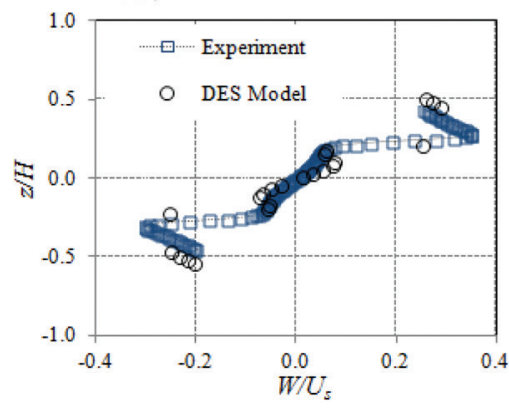

(b) $y / H=0.8, x / H=0.1$

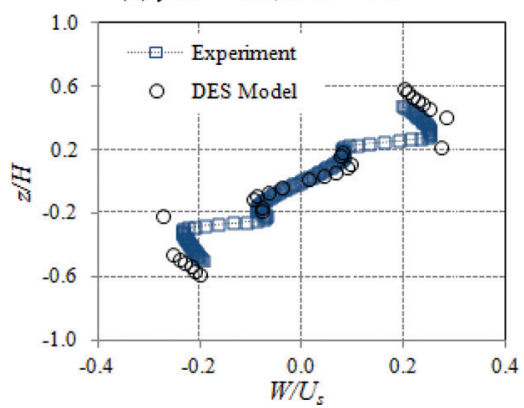

Figure 3: Computational and experimental [6-7] normalized mean transverse velocity profiles at two different locations in the wake flow [17].

using $\lambda_{2}=-1.0$. This scalar value is chosen based on the fact that it reveals the well-defined patterns in the wake flow. As shown in Figs. $4 \mathrm{~b}$ and c, the structures are clipped at two different elevations from the bed for better illustration of the fluid structures details. The horseshoe vortex structure is obvious in Fig. 4. It stretches downstream from the front of the bluff body and contributes to the three-dimensional characteristics in the wake flow. A collar vortex, embedded in a region of high vorticity in the near-bed location, emanates from the edges of 
(a) Structures zone: $0.0<y / H<1.0$

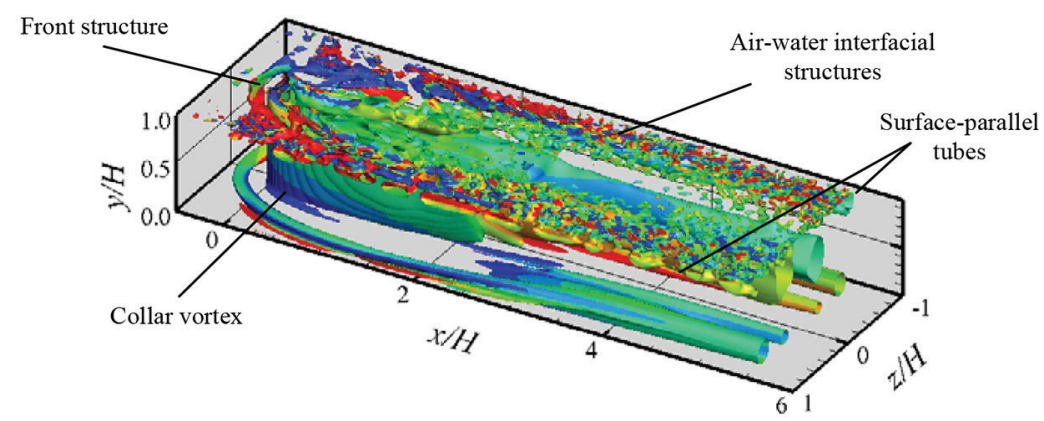

(b) Structures zone: $0.0<y / H<0.8$

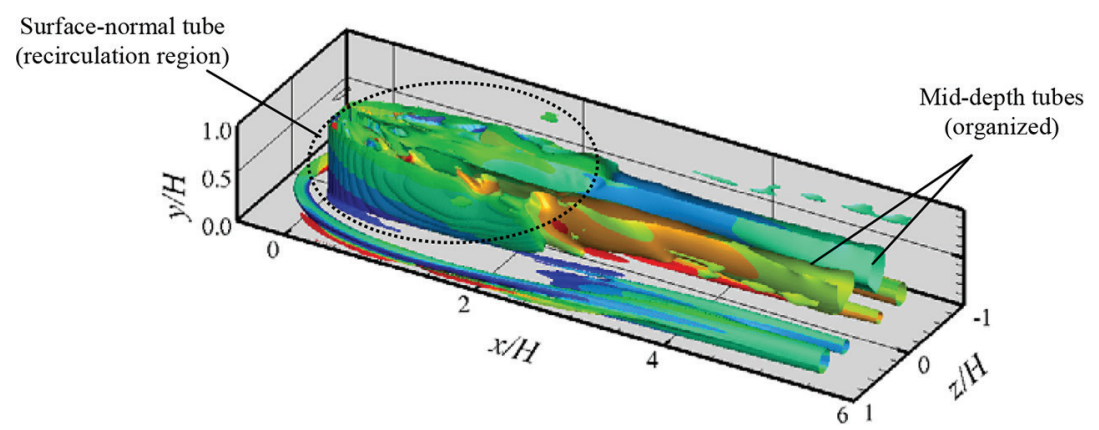

(c) Structures zone: $0.0<y / H<0.25$
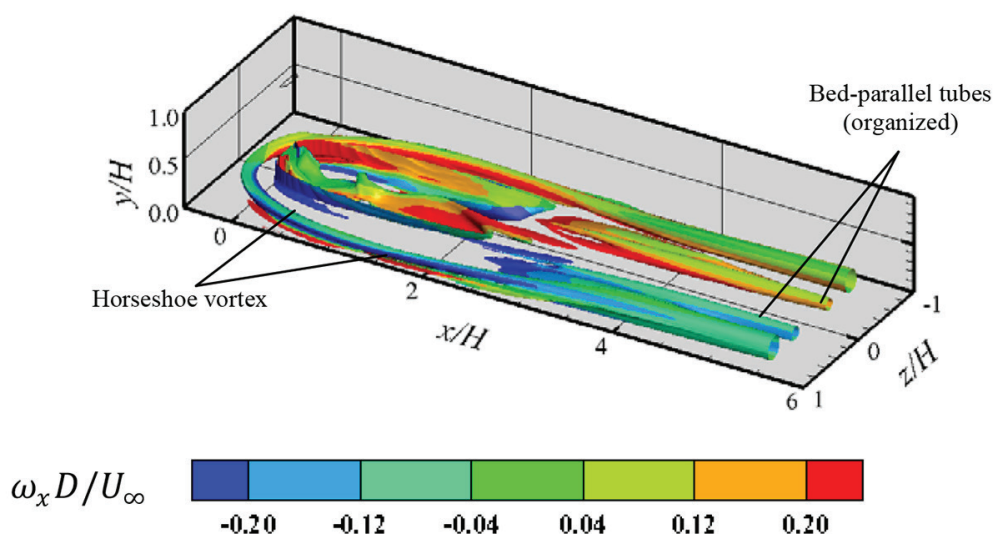

Figure 4: $\lambda_{2}$ isosurface $\left(\lambda_{2}=-1.0\right)$ for the mean flow coloured by streamwise vorticity vector component for the no-gap case.

the body. The sense of rotation of the collar vortex is similar to that of the horseshoe vortex [18]. At downstream locations $(x / H>2.0)$, two bed-parallel counter-rotating tubes (see Fig. 4c) are generated inwards next to the legs of the horseshoe vortex. These tubes reside near the bed and have the same sense of rotation as the horseshoe legs. The mechanism of formation and development of these tube structures in the mean flow is elaborated in [18-19]. The surface-normal tubes shown in Fig. $4 \mathrm{~b}$ immediately after the bluff body represent the 
averaging of the structures that shed from the sides of the bluff body in the transient flow. These structures absorb the required energy from the outer accelerating flow to overcome the wall shear stress and maintain its existence in the near-wake location. As one moves farther downstream, the accelerating flow region turns to a decelerating flow region and the kinetic energy that feeds the wake region is not sufficient to overcome the wall shear stress. Consequently, the interaction between the surface-normal tubes and the bed weakens as the kinetic energy of the accelerating flow is depleted downstream. This is the location where these structures start to detach from the bed and align with the mean flow, forming the mid-depth tubes as shown in Fig. 4b. One of the important signatures of shallow-wake flows is the existence of the surface-parallel tubes as shown in Fig. 4a. These structures are buried inside the surface layer [21] and act to transport the fluid particles from the wake core to the outer region. These structures are generated immediately underneath the free surface due to the impingement of moving fluid particles through the wake central plane with the free surface. Other minor structures, i.e. frontal structures and air-water interfacial structures were also captured at the water-air interface as shown in Fig. 4a.

Contrary to the no-gap case that is shown in Fig. 4, only traces of the horseshoe structure are sustained as the gap is introduced in the wake flow as shown in Fig. 5. The major portion of the horseshoe vortex appears to be shrunk and attenuated. This can be attributed to two different effects: on the one hand, the presence of the gap reduces the combined interaction between the approach flow, the bluff body and the bed, and therefore weakens the horseshoe structure. On the other hand, the gap flow enhances the lateral entrainment as will be explained later and acts to disintegrate and thus prevent the formation of the horseshoe structure. The effect of the gap flow is also apparent on the other turbulent structures in the wake flow as shown in Fig. 5. These structures become irregular and asymmetrical about the centre plane of the wake.

Another observation that can be drawn from Fig. 5 is that the recirculation region, immediately behind the bluff body, becomes smaller compared to the case of no gap. This is because the gap flow will sustain this region with additional kinetic energy and consequently increases the momentum and reduces the volume of this region. A new structure can also be identified in Fig. 5c, which appears near the bed, immediately after the recirculation region at the centre of the wake (blue structure). This three-dimensional structure, which is absent in the case of no gap, is stretched upwards to the vertical location $y / H=0.2$. The presence of this structure in the wake flow will enhance the wall normal velocity and accelerate the restoration of the free surface to its original level at the shorter downstream distance from the bluff body as will be investigated shortly.

Figure 6 shows the normalized wall-normal velocity $\left(V / U_{\infty}\right)$ for the two cases that are used in the current study. In this figure, the data are extracted at the centre plane of the wake. The approaching free-stream velocity, $U_{\infty}$, was used as the velocity scale for normalizing the mean velocity. The thick dotted line, marked as "free surface" at location $y / H \approx 1.0$, represents the air-water interface. A distinguishing feature of the shallow wakes is the three-dimensional roll-up structure $[18-19,22]$ between the bed and the free surface that has been observed immediately behind the bluff at the wake centre plane in Fig. 6a. This horizontally oriented structure, which is a three-dimensional spiral structure and has a positive rotation sense, plays a role to bridge both parts of the surface-normal tube (shown in Fig. 4b) and acts to transport the fluid particles from the wake core to the outer surroundings [20]. This structure does not considerably enhance the wall-normal velocity and therefore the free surface restores its original shape at a downstream location of $x / H=2.2$ from the bluff body as shown in Fig. 6a. 
(a) Structures zone: $0.0<y / H<1.0$

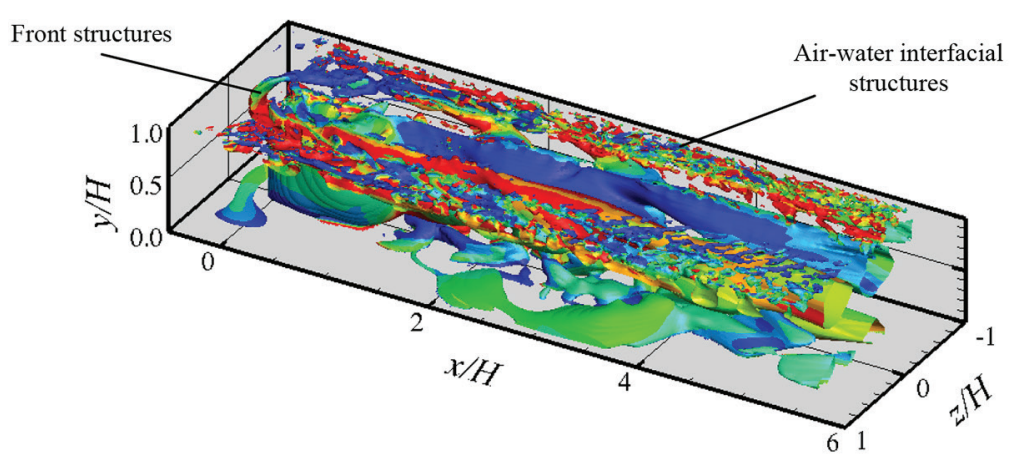

(b) Structures zone: $0.0<y / H<0.8$

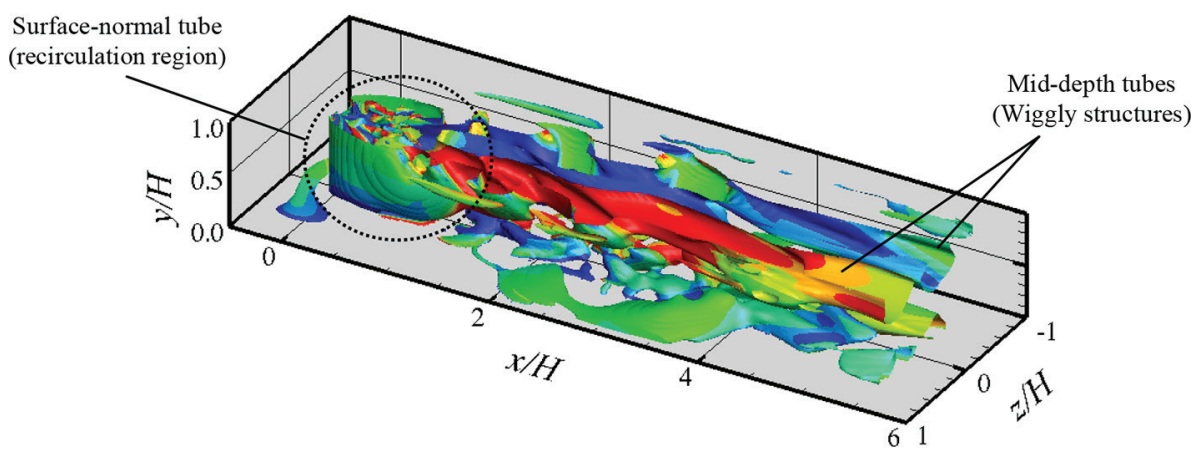

(c) Structures zone: $0.0<y / H<0.25$

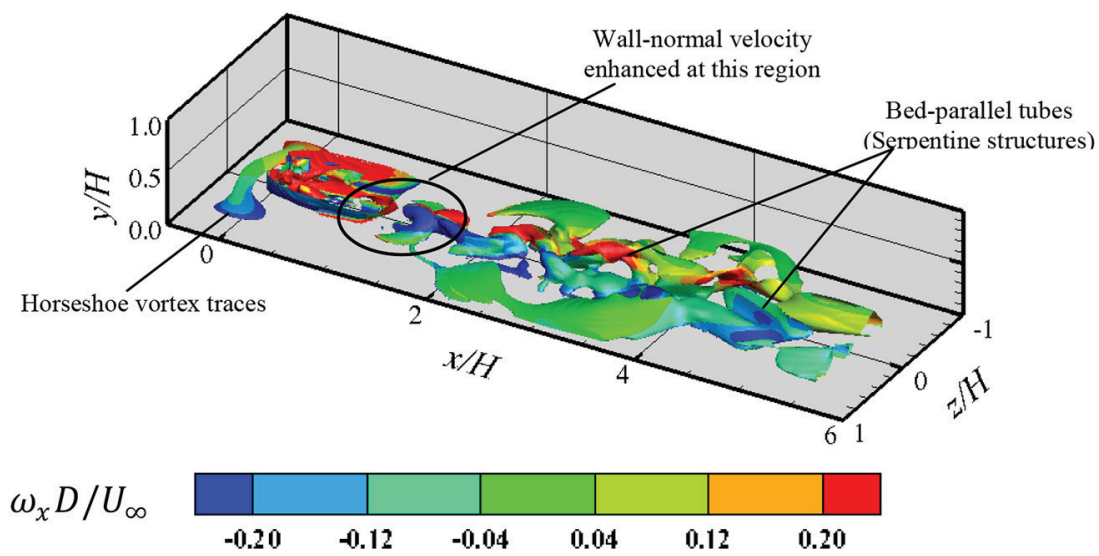

Figure 5: $\lambda_{2}$ isosurface $\left(\lambda_{2}=-1.0\right)$ for the mean flow coloured by streamwise vorticity vector component for the gap size $h / H=0$.

As the gap flow is introduced in the wake, the wall jet turns upward at a downstream distance of about $x / H=1.0$ due to the weak hydraulic jump (details are not shown here), pushing the three-dimensional roll-up structure towards the bluff body and raising it away from the bed by a dista nce equivalent to the gap height. A new structure, which has a counter rotation sense to the three-dimensional roll-up structure, will be generated near the bed at downstream 


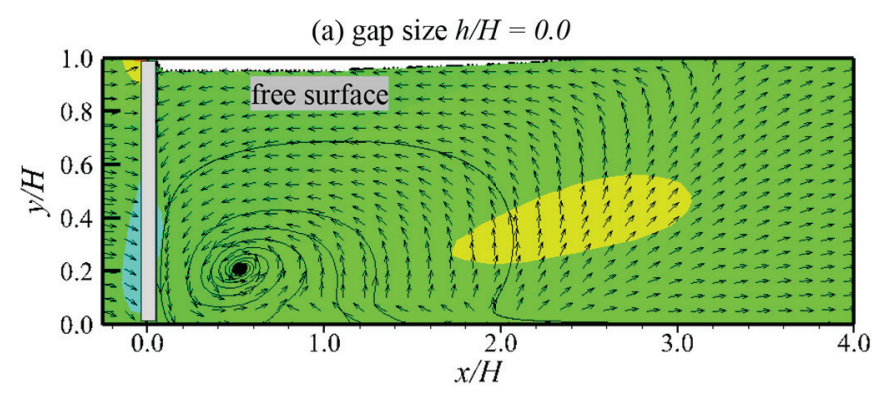

(b) gap size $h / H=0.1$

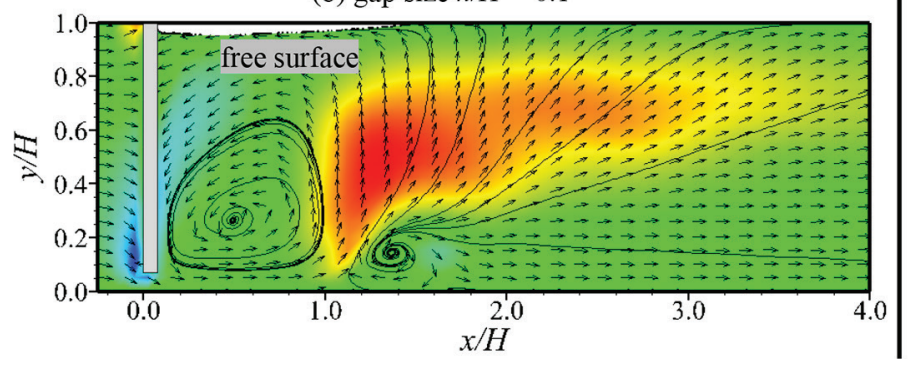

$V / U_{\infty}$

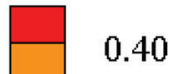

0.27

0.13

0.00

$-0.13$

$-0.27$

$-0.40$

Figure 6: Vector plot of the mean velocity field superimposed by contours of the normalized vertical velocity $\left(V / U_{\infty}\right)$ in the vertical plane $z / H=0.0$.

location $x / H=1.35$ as shown in Fig. 6b. This structure appears as a three-dimensional blue hump in Fig. 5c. The mechanism of formation of this structure is attributed to the high momentum that is associated with the wall jet, which penetrates and overcomes the reverse flow region with negative stream velocity. The strong interaction between the counter-rotating structures near the bed for the case with the gap will enhance the wall-normal velocity as shown in Fig. 6b (red contour). The wall-normal velocity enhancement occurs in the region marked in Fig. 5c. The enhancement of the wall-normal velocity in the presence of the gap explains the restoration of the free surface at shorter distances downstream of the bluff body. The free surface restores its original position at $x / H=1.4$ in the latter case as shown in Fig. $6 \mathrm{~b}$.

Figure 7 shows the contours of the normalized mean lateral velocity $\left(W / U_{\infty}\right)$ extracted at downstream locations of $x / H=0.5$ and 1.35 , respectively, for the two cases that have been investigated in the current study. The downstream distance $x / H=1.35$ has been chosen at the same location of the new structure that enhances the wall-normal velocity in the case of the gap. It is obvious from Figs. $7 \mathrm{a}$ and $\mathrm{b}$ that the concavity in the free surface in the lateral direction is comparable for either case at the downstream location $x / H=0.5$. This indicates that the gap flow has no influence on the free surface at a short distance from the bluff body. However, the effect of the gap flow is apparent on the free surface at downstream location $x / H=1.35$ as shown in Figs. $7 \mathrm{c}$ and d. The depression in the free surface spans in the lateral direction between $-1.2<z / H<1.2$ as shown in Fig. 7c, while the free surface approximately restored its origin position at $y / H=1.0$ for the case with the gap as illustrated in Fig. 7 d.

In light of the above discussion (see Figs. 5-7), one can conclude that the free surface starts to restore its original shape at approximately a downstream location $x / H=1.0$, a location of the interaction between the new structure that is generated due to the gap flow and three-dimensional roll-up structure as shown in Fig. 6b. Another conclusion which can be drawn from Fig. 7 is that 


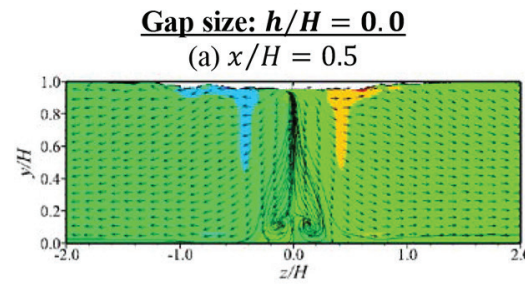

(c) $x / H=1.35$

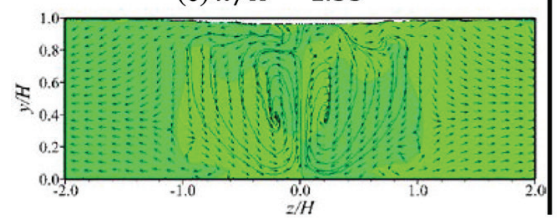

$W / U_{\infty}$

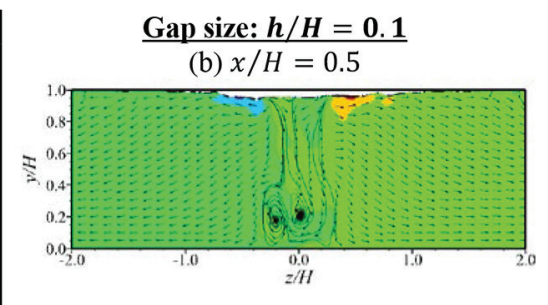

(d) $x / H=1.35$

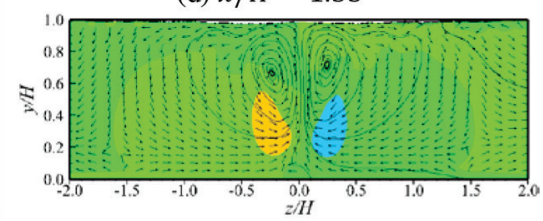

Figure 7: Vector plot of the mean velocity field superimposed by contours of the normalized transverse velocity $\left(W / U_{\infty}\right)$ in the vertical plane.

the gap flow enhances the entrainment at downstream location $x / H=1.35$, i.e. beyond the locations where the enhancement of the wall-normal velocity occurs, as shown in Fig. 7d. The wall-normal velocity will increase the inward transverse flow since it entrains more fluid into the wake region at these regions. This observation agrees with the experimental investigations that were performed by Shinneeb and Balachandar [6]. The gap flow has a slight effect on the lateral entrainment at the vertical plane $x / H=0.5$ as shown in Fig. $7 \mathrm{~b}$. However, the wall jet will affect and scramble the topological feature of the two spiral structures (two foci) on the sides of the wake centre plane and lifts them a bit from the bed in the near-wake region. The enhancement in the wall-normal velocity and the lateral velocity for the case of the gap will also affect the topological feature of the structures in the far-wake region (details are not shown here). The degree of randomness of the structures increases as one moves further downstream.

\section{CONCLUSION}

The present study is oriented towards an investigation of the influence of the gap in a wake on the flow characteristics, fluid structures and the free surface restoration. The numerical study was carried out using the finite volume technique. The fluid structures were identified using the $\lambda_{2}$-criterion which successfully distinguishes the rotational contribution of the vorticity distribution. The following results are concluded from the current study:

- A new structure at the near-bed location is generated when the gap is introduced in the wake flow. The interaction between this structure and the adjacent structures will enhance the wall-normal component velocity and restore the free surface to its original shape at a shorter downstream location in the presence of the gap case.

- One of the consequences of the gap is to reduce the size of the wake. The gap flow will sustain this region with additional kinetic energy and consequently reduces the wake size.

- The lateral entrainment from the surroundings to the wake region increases as the gap is introduced in the wake flow. This can be attributed to the increase of the magnitude of the positive wall-normal velocity component which is enhanced by the gap flow. 
- As the gap is introduced in the wake flow, the fluid structures become irregular and asymmetrical about the centre plane of the wake. The degree of randomness increases in the far-wake region.

- The major portion of the horseshoe vortex appears to be intermittent and attenuated as the gap is introduced in the wake.

\section{REFERENCES}

[1] Dey, S., Lodh, R. \& Sarkar, S., Turbulence characteristics in wall-wake flows downstream of wall-mounted and near-wall horizontal cylinders. Environmental Fluid Mechanics, 18(4), pp. 1-31, 2018.

[2] Panigrahi, P.K., PIV investigation of flow behind surface mounted detached square cylinder. Journal of Fluids Engineering, 131(1), 011202, 2009.

[3] Wang, X.K. \& Tan, S.K., Experimental investigation of the interaction between a plane wall jet and a parallel offset jet. Experiments in Fluids, 42(4), pp. 551-562, 2007.

[4] Martinuzzi, R.J., Bailey, S.C.C. \& Kopp, G.A., Influence of wall proximity on vortex shedding from a square cylinder. Experiments in Fluids, 34(5), pp. 585-596, 2003.

[5] Taniguchi, S. \& Miyakoshi, K., Fluctuating fluid forces acting on a circular cylinder and interference with a plane wall. Experiments in Fluids, 9(4), pp. 197-204, 1990.

[6] Shinneeb, A.M. \& Balachandar, R., Effect of gap flow on the shallow wake of a sharpedged bluff body-mean velocity fields. Journal of Turbulence, 17(1), pp. 94-121, 2016.

[7] Shinneeb, A.M. \& Balachandar, R., Effect of gap flow on the shallow wake of a sharpedged bluff body-turbulence parameters. Journal of Turbulence, 17(1), pp. 122-155, 2016.

[8] Shinneeb, A.M., Balachandar, R. \& Zouhri, K., Effect of gap flow on the shallow wake of a sharp-edged bluff body_Coherent structures. Physics of Fluids, 30(6), 065107, 2018.

[9] CD-adapco, STAR-CCM+ V10.06.009, User Manual, 2015.

[10] Hirt, C.W. \& Nichols, B.D., Volume of fluid (VOF) method for the dynamics of free boundaries. Journal of Computational Physics, 39(1), pp. 201-225, 1981.

[11] Muzaferija, S., A two-fluid Navier-Stokes solver to simulate water entry. In Proceedings of 22nd Symposium on Naval Architecture, pp. 638-651, 1999.

[12] Leonard, B.P., The ULTIMATE conservative difference scheme applied to unsteady one-dimensional advection. Computer Methods in Applied Mechanics and Engineering, 88(1), pp. 17-74, 1991.

[13] Spalart, P.R., Comments on the feasibility of LES for wings, and on a hybrid RANS/LES approach. In Proceedings of First AFOSR International Conference on DNS/LES, 1997.

[14] Wilcox, D.C., Simulation of transition with a two-equation turbulence model. AIAA Journal, 32(2), pp. 247-255, 1994.

[15] Wilcox, D.C., Turbulence Modeling for CFD. La Canada, CA: DCW Industries, Vol. 2, pp. 103-217, 1998.

[16] Hoffmann, K. \& Chiang, S., Computational Fluid Dynamics, Vol. 3, 4th ed., Engineering Education System, Wichita, KS, USA, 2000.

[17] Nasif, G., Balachandar, R. \& Barron, R.M., Influence of bed proximity on the threedimensional characteristics of the wake of a sharp-edged bluff body. Physics of Fluids, 31(2), p. 025116, 2019.

[18] Nasif, G., Barron, R.M. \& Balachandar, R., DES evaluation of near-wake characteristics in a shallow flow. Journal of Fluids and Structures, 45, pp. 153-163, 2014. 
[19] Nasif, G., Balachandar, R. \& Barron, R.M., Characteristics of flow structures in the wake of a bed-mounted bluff body in shallow open channels. Journal of Fluids Engineering, 137(10), 101207, 2015.

[20] Nasif, G., Balachandar, R. \& Barron, R.M., Mean characteristics of fluid structures in shallow-wake flows. International Journal of Multiphase Flow, 82, pp. 74-85, 2016.

[21] Maheo, P., Free Surface Turbulent Shear Flow, PhD dissertation, California Institute of Technology, CA, USA, 1999.

[22] Akilli, H. \& Rockwell, D., Vortex formation from a cylinder in shallow water. Physics of Fluids, 14(9), pp. 2957-2967, 2002. 